\title{
Wenn Rheuma sich nicht an Organgrenzen hält
}

\begin{abstract}
Meist sind es die Muskeln oder die Gelenke, an denen sich entzündlich-rheumatische Erkrankungen zuerst bemerkbar machen. Bei vielen Patienten greifen die rheumatypischen Entzündungen jedoch auch auf andere Organe und Gewebe über und verursachen Begleiterkrankungen, die prinzipiell den ganzen Körper betreffen können. Wie häufig diese sind, vor welche Herausforderungen sie Ärzte und Patienten stellen und wie die Versorgung dieser Patienten verbessert werden kann, war ein Thema auf dem Online-Jahreskongress der Deutschen Gesellschaft für Rheumatologie (DGRh).
\end{abstract}

„Das eine“ Rheuma gibt es nicht. Unter dem Begriff der entzündlich-rheumatischen Erkrankungen werden sehr unterschiedliche Krankheitsbilder zusammengefasst, deren Gemeinsamkeit eine Fehlsteuerung des Immunsystems ist. Hierdurch kommt es zu Angriffen auf körpereigenes Gewebe und in der Folge zu chronischen Entzündungen. „Weil Immunzellen und ihre Botenstoffe frei im Körper zirkulieren, können prinzipiell alle Körperbereiche von der Entzündung betroffen sein“, sagt Prof. Andreas Krause, Vorstandsmitglied der DGRh und Chefarzt am Immanuel Krankenhaus Berlin. Wie Daten aus der Kerndokumentation des Deutschen Rheumaforschungszentrums zeigen, sind rund $80 \%$ der Patienten mit klassischem Gelenkrheuma von mindestens einer Begleiterkrankung betroffen, bei anderen rheumatischen Erkrankungen liegt die Zahl ähnlich hoch.

Begleiterkrankungen wie Herz-KreislaufBeschwerden, Lungenerkrankungen oder Depressionen haben einen erheblichen Einfluss auf die Lebensqualität der Patienten, den Krankheitsverlauf und die Wirksamkeit der Medikation. „Es wird deutlich, dass Rheumatologen internistisch ausgebildet sein sollten, denn dies ist die notwendige Basis, um bei der Therapie die Begleiterkrankungen mitberücksichtigen zu können“, sagt Krause. „Dazu gehört auch, dass aktiv nach ihnen gesucht wird, denn nur dann lässt sich frühzeitig gegensteuern“. Nicht alle Erkrankungen seien jedoch gleichermaßen im Bewusstsein der behandelnden Ärzte präsent: Während das erhöhte 
Osteoporose- oder Infektionsrisiko von Rheumapatienten in der Regel berücksichtigt werde, sei das bei anderen Komorbiditäten wie Herz-Kreislauf-Erkrankungen oder Depressionen oft nicht der Fall.

Auch eine Lungenbeteiligung wird oft lange nicht erkannt. Von großer Bedeutung sind hier die sog. interstitiellen Lungenerkrankungen (ILD), die das Binde- und Stützgewebe der Lunge betreffen und es durch die permanente Entzündung vernarben lassen. Hierdurch geht gesundes Lungengewebe verloren, mit der Zeit leiden die Patienten an Kurzatmigkeit und Husten, und in 10-20\% der Fälle tragen die Lungenschäden auch zum vorzeitigen Tod der Patienten bei. Interstitielle Lungenveränderungen treten oft schon sehr früh im Krankheitsverlauf auf, verursachen aber lange Zeit keine Beschwerden. „In ComputertomographieAufnahmen finden sich bei $60 \%$ der Gelenkrheuma-Patienten Anzeichen für eine ILD, klinische Symptome allerdings nur bei $6 \%$ “, sagt Krause. Daher sollten Patienten mit Rheumatoider Arthritis bereits bei der Erstdiagnose und dann in regelmäßigen $A b$ ständen wieder auf eine ILD untersucht werden.

„Auch um andere Begleiterkrankungen frühzeitig erkennen und behandeln zu können, sind sorgfältige Vorsorgeuntersuchungen notwendig“, mahnt auch DGRh-Kongresspräsident und -Vorstandsvorsitzender Prof. Hendrik Schulze-Koops vom Klinikum der Universität München. Die hierfür notwendige Zeit stehe aufgrund des rheumatologischen Fachärztemangels jedoch häufig nicht zur Verfügung. Kurzfristige Abhilfe könnten einfache, wenig zeitaufwändige Screeningmethoden schaffen, die eventuell auch vom nicht-ärztlichen rheumatologischen Assistenzpersonal übernommen werden könnten, so die DGRh. Auf Dauer sei eine angemessene Versorgung von Patienten mit Begleiterkrankungen jedoch nur möglich, wenn die Zahl internistischrheumatologischer Fachärzte deutlich gesteigert werde.

Nach einer Pressemitteilung der Deutschen Gesellschaft für Rheumatologie 